\title{
Some Aspects of Galactic Cosmic Ray Acceleration
}

\author{
Yousaf Mahmood Butt \\ Harvard-Smithsonian Center for Astrophysics, 60 Garden St., \\ Cambridge, MA, USA
}

\begin{abstract}
I give a synopsis of two aspects of the Galactic Cosmic Ray (GCR) acceleration problem: the importance of the medium energy gamma-ray window, and several specific astrophysical sources which merit further investigation. NOTE: figures may be found in the on-line version only: astro-ph/0309758.
\end{abstract}

\section{INTRODUCTION}

Cosmic rays continually rain down on our heads, but we have virtually no better idea where they come from than did investigators fifty years ago (eg. Shklovskii, 1953). Gamma-ray astronomy has been the tool of choice to investigate their origin(s) since a local overdensity of freshly accelerated GCRs are expected to produce co-spatial high energy (gamma-ray) photons. Of course, the proper interpretation of such gamma-ray data (specifically, whether electrons or nuclei are generating the detected radiation) requires as broad a multi-wavelength coverage as practical: gamma-ray data is necessary but not sufficient to prove the existence of a GCR acceleration site. Unfortunately, sufficient multi-wavelength coverage of requisite fidelity is often simply unavailable. As outlined by Francis Halzen in this session, now neutrino astronomy also appears poised to contribute to GCR research in the 'non-wavelength' window.

Despite almost 40 years of concerted effort in gamma-ray astronomy, not a single source of nucleonic GCRs has yet been firmly identified. The main reason for this is the rather marginal spatial resolution of virtually all gammaray instrumentation flown, which results in severe source confusion - eg. are the gamma-rays coming from a Supernova Remnant (SNR) shock or a related, or unrelated, pulsar? The latest ground-based stereo Cherenkov telescopes and the next-generation orbiting $\mathrm{GeV}$ detectors promise to finally overcome this limitation.

Currently, there are two favored theoretical mechanisms for accelerating GCRs, and both invoke shocks: either the shocks of isolated SNRs in the $\sim 10^{4}-10^{5}$ yrs after explosion (eg. Drury et al., 2001; see Torres et al., 2003 for a recent review and Plaga, 2002 for an alternative hypothesis); or, the cumulative shocks of massive stars and/or multiple SNRs in massive stellar associations (or after evolution, 'superbubbles') which last $\sim 10^{7} \mathrm{yrs}$ (eg. Cesarsky \& Montmerle, 1983; Bykov 2001). It is certainly also possible that both mechanisms could be operating simultaneously in the Galaxy. Although as a class only SN appear to have the required power input to the ISM to explain the local energy density of GCRs, it is unclear whether the physical mechanism for accelerating nucleonic GCRs requires that multiple SNRs be embedded in a superbubble or 
stellar cluster. Interestingly, although the bulk of SN are expected to occur in superbubbles, many more individual, isolated, SNRs have been identified and cataloged than superbubbles.

\section{THE IMPORTANCE OF THE MeV WINDOW}

Although the acceleration of high energy CRs in a given source cannot be proven without the detection of similarly high energy ( $\mathrm{GeV}-\mathrm{TeV}$, and even higher energies) radiation localized with the source, the lower-energy $\mathrm{MeV}$ window is still very important in discriminating the nature of the particles generating the detected gamma-rays: electrons vs. nuclei. This is because in the case of a hadronic origin there is a plateau expected in the gamma-ray spectrum in the approx. 1$100 \mathrm{MeV}$ range. Such a discontinuous 'plateau-ing' of the photon spectrum is not expected from purely leptonic bremsstrahlung or inverse-Compton emissions. The inability to distinguish the nature of the particles generating the detected gamma-rays has been a major stumbling block in nucleonic GCR origin studies (eg. see Reimer \& Pohl, 2002 and Butt et al., 2002). High signal-to-noise data in the soft-gamma window greatly simplifies our ability to assign a hadronic vs. leptonic origin to the detected gamma-rays (Fig. 1; see on-line version, astro$\mathrm{ph} / 0309758$, for all figures). This is a direct consequence of characteristic shape of the hadronic pion-decay spectrum: in fact, were it not for the existence of, and emission from, hadronically-generated secondary electrons, we would expect a highly distinctive, symmetrical pion 'hump' centered at $67.5 \mathrm{MeV}$ (=half the pion rest mass) in the case that hadrons were producing the gamma-rays (eg. Schlickeiser 1982). Note that in Schlickeiser's paper a steady-state leptonic population was assumed so that the expected plateau in SNRs/stellar associations' hadronic emission will be even more distinctive than shown in that study as steady-state has not been reached in the typical lifetimes of those objects, and thus less secondaries are present. Though the INTEGRAL satellite covers the 1$10 \mathrm{MeV}$ bandpass with relatively good spatial resolution, there is unfortunately a gap in the important approx. 10-100 $\mathrm{MeV}$ range. (The $>50 \mathrm{MeV}$ bandpass will be covered by AGILE and GLAST instruments in the near future.) Orbiting detectors, such as the proposed MEGA instrument (Kanbach et al., 2003) would thus be very useful for GCR studies by filling-in this bandpass gap.

\section{SPECIFIC SOURCES}

Below I provide an incomplete list of sources which would be useful to observe in gamma-rays as well as other wavelengths. I have divided the sources into three categories: isolated SNRs, stellar clusters/superbubbles, and extragalactic starburst galaxies. There are many other sources worth investigating: the list is not exhaustive.

I. Isolated Galactic Supernova Remnants (SNRs)

a. G347.3-0.5 (RX J1713.7-3946)

The SNR G347.3-0.5 (RX J1713.7-3946) and its environs are amongst the best laboratories for investigating shock-driven particle acceleration processes in our Galaxy. Photons spanning 17 orders of magnitude in frequency, from radio through $\mathrm{TeV}$ range, have been reported as being plausibly associated with this 
SNR, or with its putative shock-molecular cloud interactions (Fig 2). Deeper radio observations of this source would help pin down the contributions of electrons vs. nucleons to the gamma-ray emission. High resolution $\mathrm{mm}$ observations of $1 \rightarrow 0,2 \rightarrow 1$, and higher rotational level transitions of $\mathrm{CO}$ would help better determine the cloud excitation, and possibly, also whether the cloud shape 'meshes' with the SNR shock front. Similarly, high spatial resolution (CHANDRA) X-ray observations are needed towards the $\mathrm{N}$ of the remnant to image whether indeed the shock front and clouds are interacting. This is important in settling whether the clouds and the SNR are interacting, which is the key in determining the distance to the SNR. (The distance to the SNR is currently assumed be the same as the clouds with which it is thought to be interacting). Gamma-ray observations would also help narrowing down the rather large EGRET error box associated with this source. Specifically, one would like to know whether gamma-ray emission is consistent with the location of the molecular clouds, and whether it's spectrum agrees with a hadronic origin. The recent report of a hard ASCA source (AX J1714.1-3912) seen immediately adjacent to the SNR rim (Uchiyama et al., 2002) and superposed with 'cloud A' lends particular urgency and significance to multi-wavelength observations of this highly non-thermal SNR.

b. The SNRs W28 (G6.4-0.1); W44 (G34.7-0.4); and W66 (gamma-Cygni, G78.2+2.1) All three of these SNR-EGRET gamma-ray source coincidences have been discussed extensively in the literature, and are reviewed in detail by Torres et al. (2003). These SNRs all appear to be in interaction with neighboring massive molecular clouds which may explain the detected gamma-ray emissions; however, all three gamma-ray sources are also in coincidence with energetic pulsars/compact objects which could, alternatively - or, in addition - be generating the gamma-rays. Similar figures as Fig. 2 above for all three cases are available in Torres et al. (2003) [see Fig.'s 21, 22, 25, 28 therein], and are not reproduced here in the interest of brevity. Even before AGILE and GLAST are launched, INTEGRAL observations of these SNRs are needed to better localize the gamma-ray emission region(s). Such data will simultaneously greatly aid in clarifying the nature (hadronic vs. leptonic) of the emissions in these $3 \mathrm{im}$ portant SNRs by providing a MeV spectrum (eg. see Fig. 1). Although not detected in the $\mathrm{TeV}$ range with the previous generation of Cherenkov telescopes, deeper observations with the updated arrays would be of great use.

c. RCW 86 (MSH 14-63, G315.4-2.3)

RCW 86 is a bright shell-like SNR with non-thermal X-ray and radio emission which is especially strong towards the SW, where there is also evidence of interaction with a molecular cloud (eg. Borkowski et al., 2001; Rosado et al., 1996). In fact, the intensity of the the non-thermal X-ray emission from the SW shell is even brighter than that of SN 1006. RCW 86 also appears to be associated with a neighboring $\mathrm{OB}$ association (Westerlund, 1969). Very recently the CANGAROO collaboration has reported the detection (at the approx. 4 sigma level) of $\mathrm{TeV}$ range gamma-rays from the non-thermal X-ray bright southwest shell at a level of $\sim(3 \pm 0.7) 10^{-12}$ photons $\mathrm{cm}^{-2} \mathrm{sec}^{-1}$ (Watanabe et al., 2003). Vink et al. (2000) and Gvaramadze \& Vikhlinin (2003) also report the presence of point-like X-ray sources towards the same SW shell. Data in the INTEGRAL wave band, and at higher energies, of this SNR will be useful to test whether 
there is associated $\mathrm{MeV}$-range gamma-ray emission from the SW shell; again, the spectrum of such putative emission will greatly aid in discriminating the origin of the high-energy flux. Due to their high spatial resolution, JEM-X (and IBIS) data will be especially useful in examining whether it is the point-like sources in the SW or rather the extended shock there that is generating the gamma-rays.

\section{d. Monogem Ring SNR/PSR B0656+14}

The Monogem Ring is a very large ( 20 degree diameter), bright SNR with a young radio pulsar PSR B0656+14 projected virtually at its geometric center (see Fig. 1 in Thorsett et al., 2003 for an X-ray mosaic image). Very recently, Thorsett et al. (2003) have convincingly argued that the pulsar and the SNR are indeed related (contrary to some previous studies), and that this SNR - by itself - may be responsible for the existence of the 'knee' in the GCR spectrum at $\sim 3 \mathrm{PeV}\left(310^{15} \mathrm{eV}\right)$. Indeed, Erlykin \& Wolfendale $(1997,2003)$ already theorized that a single SNR of age 100kyr and 325pc from earth could by itself explain the knee feature - these parameters agree surprisingly well with those of the Monogem Ring SNR. In contrast to the previously mentioned SNRs in this presentation there is no high-energy EGRET source or reports of $\mathrm{TeV}$ emission associated with this SNR. However, this may not be surprising given its large size which would tend to 'wash-out' the signal due to the integrated background over the large SNR region.

II. Galactic OB Associations/Superbubbles

a. Cygnus OB2

Cyg OB2 is the most massive OB association known in the Galaxy, thought to contain in excess of $2500 \mathrm{OB}$ type stars (Knoldseder 2002). Indeed, Hanson (2003) mentions several new massive star members in this association which were previously unknown due to the heavy extinction in the Cygnus direction. Recently, the HEGRA Chrenkov array group has reported a steady and extended TeV-range gamma-ray source within this association ( TeV J2032+4032; Aharonian et al., 2002). An EGRET source, 3EG 2033+4118, and a GeV source are coincident with, and nearby, the $\mathrm{TeV}$ source, respectively. We have carried out preliminary CHANDRA and VLA observations of this intriguing $\mathrm{TeV}$ source and have argued that the most likely explanation is that nuclei (rather than electrons) accelerated to high energies are responsible for the gamma-ray emissions (Butt et al., 2003). If confirmed by future observations, such a scenario would strongly support a stellar association/superbubble origin of GCRs, as has been theoretically argued for some time (eg. Cesarsky \& Montmerle, 1983; Bykov, 2001; and Parizot, 2002).

b. RCW 38 \& NGC3603

I group these two stellar clusters together since recent CHANDRA observations have found evidence for hard, diffuse $\mathrm{X}$-ray emission associated with both. Diffuse non-thermal X-ray emission from the southern stellar cluster RCW 38 was found in a recent $100 \mathrm{ksec}$ CHANDRA observation (Wolk et al., 2002). This is one of the brightest HII regions at radio wavelengths (eg. Wilson et al., 1970 ), and is a natural candidate for deeper multi-frequency observations. The $\mathrm{X}$-ray emission fills the center of a radio ring, reminiscent of some shell-type SNRs (Fig 3) and is very intriguing in the context of a young cluster/HII region. If hard X-ray/gamma-ray emission is discovered superposed to the non-thermal 
$\mathrm{X}$-rays, this would be a very significant result and would argue strongly in favor of collective shocks from the young stars accelerating GCRs to very high energies as proposed by, eg., Cesarsky \& Montmerle (1983). (The age of the cluster is too young for there to be any 'contaminating' contributions from SNRs).

NGC 3603 is among the most massive and luminous visible starburst regions in the Galaxy. A recent 50 ksec CHANDRA observation has similarly found hard diffuse X-ray emission spatially coincident with the cluster core (Moffat et al., 2002). Those authors attribute this emission to the collective effects induced by the multiple, colliding stellar winds from the large population of massive stars in the region. As in the case of RCW38, if future observations find significant hard X-ray/gamma-ray emission then this would be a major result since it would again favor the collective stellar wind hypothesis of the origin of (at least some fraction of) GCRs. CHANDRA has also detected diffuse X-ray emission from the Omega (M17/W38) \& Rosette Nebulae (NGC2237-2246) but since the emission is soft (Townsley et al., 2003 \& Dunne et al., 2003), we do not anticipate that these objects are significant GCR accelerators.

III. The Starbursts NGC 253 \& M82 (NGC 3034)

NGC 253 and M82 are amongst the brightest IR galaxies and are considered the prototypical starbursts. At 2.6 Mpc NGC 253 is also one of the closest. The OSSE spectrometer aboard CGRO has already positively detected continuum emission from NGC 253 up to $165 \mathrm{keV}$ (Bhattacharya et al., 1994) and very recently the CANGAROO Cherenkov telescope collaboration has also reported a detection of this source in TeV-range gamma-rays (Itoh et al., 2003a). Although those authors have favored an explanation in terms of electronic IC emission (Itoh et al., 2003b), others have provided an alternative theory: that the high energy emission may be due to nucleonic cosmic rays accelerated to high energies via the collective effects engendered by the starburst activity (Romero \& Torres, 2003; see also Völk, 2003; and Sugai et al., 2003). In effect, these starbursts can be considered simply scaled-up versions of Galactic HII regions mentioned in the previous section. The goal of further multi-wavelength (in particular, gammaray) observations would be to confirm the soft gamma-ray emission from these starbursts and, in combination with future AGILE and GLAST datasets, to resolve the question over the type of particles producing the emission.

At 3.2 Mpc M82 is only slightly further away than NGC 253, and this alone may have been the cause of its non-detection in the OSSE datasets until 1994 (Battacharya et al., 1994). Note that M82 was detected by the HEAO A4 detector (Gruber \& MacDonald, 1993), and future multi-wavelength studies are suggested.

This brief review benefited from discussions with A. Bykov, P. Benaglia, J. Combi, T. Dame, J. Drake, Ph. Durouchoux, M. Kaufman Bernad, P. Milne, F. Miniati, M. Pohl, O. Reimer, J. Rodriguez, G. Romero, M. Rupen, D. Torres, and S. Wolk. The support of the CHANDRA project, NASA Contract NAS839073 , is gratefully acknowledged.

\section{References}

Aharonian, F., et al., 2001, A\&A, 370, 112

Aharonian, F., et al., 2002, A\&A, 393L, 37 
Bhattacharya, D., et al., 1994, ApJ, 437, 173

Butt, Y., et al., 2002, Nature 418, 499

Butt, Y., et al., 2003, ApJ, accepted, astro-ph/0302342

Bykov, A., 2001, SSRv, 99, 317

Borkowski, A., et al., 2001 ApJ, 550, 334

Cesarsky, C., \& Montmerle, T., 1983, SSRv, 36, 173

Drury, L., et al., 2001, SSRv, 99, 329

Dunne, B., et al., 2003, ApJ, 590, 306

Erlykin, A., \& Wolfendale, A., 1997, J. Phys. G, 23, 979

Erlykin, A., \& Wolfendale, A., 2003, J. Phys. G, 29, 709

Gruber, D., \& MacDonald, 1993, priv. comm. as plotted in Figure 2 of Bhattacharya et al., 1994

Gvaramadze, V. V., \& Vikhlinin, A. A., 2003, A\&A, 401, 625

Hanson, M., 2003, ApJ in press, astro-ph/0307540

Itoh, C., et al., 2003a A\&A, 402, 443I

Itoh, C., et al., 2003b, ApJ Lett, submitted, astro-ph/0301147

Kanbach, G., et al., 2003 (SPIE) X-Ray and Gamma-Ray Telescopes and Instruments for Astronomy. Edited by Joachim E. Truemper, Harvey D. Tananbaum. Proceedings of the SPIE, Volume 4851, pp. 1209-1220

Knodlseder, J., 2003 proceedings of the IAU Symp 212, A Massive Star Odessey from Main Sequence to Supernova, in press

Moffat, A, et al., 2002, ApJ, 573, 191

Plaga, R., 2002, New Astronomy, 7, 317

Parizot, E., 2002, in the Proceedings of the XXXVIIth RENCONTRES DE MORIOND meeting, Les Arcs, March 9-16, 2002.

Plaga, R., 2002, New Astronomy, Volume 7, Issue 6, p. 317

Reimer, O., \& Pohl, M., 2002, A\&A, 390L, 43

Romero, G. \& Torres, D., 2003, ApJ, 586L, 33

Rosado, M., et al., 1996, A\&A, 315, 243

Schlickeiser, R., et al., 1982, A\&A, 106L, 5

Shklovskii, I. S., 1953, Dokl. Akad. Nauk SSSR, 91, No 3, 475-478 (1953) [Library of Congress Translation n. RT-1495]

Slane, P., et al., 1999, ApJ, 525, 357S

Sugai et al., 2003, ApJ, 584L, 9

Thorsett, S. E., et al., 2003, ApJ, 592L, 71

Torres, D., et al., 2003, Physics Reports, Vol. 382, No. 6, August 2003

Townsley, L., et al.,2003, ApJ in press, astro-ph/0305133

Uchiyama, Y., et al., 2002, PASJ, 54L, 73

Vink, J., et al., 2000, A\&A, 362, 711

Volk, H., 2003 Invited paper presented at "The Universe Viewed in Gamma-Rays" held on 25-28 Sept. 2002 in Kashiwa Japan, astro-ph/0303078

Watanabe, J., et al., 2003, Proceedings of the 28th ICRC, pp. 2397-2400, in press

Westerlund, B. E., 1969, AJ, 74, 879

Wilson, T. L., et al., 1970, A\&A, 6, 364

Wolk, S. et al., 2002, ApJ, 580L, 161 